\section{Nivel sérico de folato $y$ vitamina $B$ en adultos mayores chilenos. Resultados de la Encuesta Nacional de Salud ENS 2009-2010}

\author{
CECILIA CASTILLO-LANCELLOTTI ${ }^{1}$, PAULA MARGOZZINI ${ }^{2}$, \\ GONZALO VALDIVIA ${ }^{2}$, OSLANDO PADILLA ${ }^{2}$, RICARDO UAUY ${ }^{3,4}$, \\ JAIME ROZOWSKI ${ }^{5}$, JOSEP A. TUR ${ }^{1,6}$
}

\section{Serum folate and vitamin $B_{12}$ in older people. Results from the Chilean national health survey 2009-2010}

Background: Supraphysiological levels (SFL) of serum folate (SF) derived from flour fortification with folic acid (FA) could be risky among older adults with low vitamin B12 (B12) levels. Aim: To describe and analyze SF and B12 levels in older Chileans and to identify risk groups. Material and Methods: Participants were 1.043 older people aged $\geq 65$ years from the National Health Chilean Health Survey 20092010 (ChNHS 2009-10), a multistage stratified random sample, representative of the national population. SF $(\mu \mathrm{g} / \mathrm{L})$ and $B 12(\mathrm{pg} / \mathrm{ml})$ were determined in fasting samples by competitive chemoluminescence immunoassay. Mean, deciles and percentiles 5 and 95th were calculated. We defined SF categories: $<4.4$ (deficit); 4.41-20 (normal) and SFL: 20.01-25.6; 25.6-29 and $>29 \mu \mathrm{g} / \mathrm{L}$ (80th percentile of the distribution) and vitamin B12 categories: $\leq 200$ (deficit); 200.1-299.5 (marginal deficit) and $>299.5$ (normal). Prevalence rates, multiple and logistic regression models were used and adjusted by sex and age, educational level and residence area. Results: SF and B12 mean and 95th percentiles were $21.2 \pm 0.56 / 38.6 \mu \mathrm{g} / \mathrm{L}$ and $348.4 \pm 7.6 / 637(\mathrm{pg} / \mathrm{ml})$ respectively. Forty nine percent of participants had SFL. Folate and B12 deficiency were present in 0.3 and $8.1 \%$ of participants, respectively. Men had significantly lower prevalence of $S F L>29 \mu \mathrm{g} / \mathrm{L}$ (OR adjusted odds ratio $0.4795 \%$ confidence intervals: 0.26-0.84). B12 showed no significant variation by age and sex. The prevalence of SFL associated with B12 deficiency was $4.1 \%$. No statistically significant association was observed between levels of folate and B12. Conclusions: Folate deficit is almost inexistent, but a significant percentage of participants had SFL suggesting the need for revising the current wheat flour fortification levels.

(Rev Med Chile 2013; 141: 1107-1116).

Key words: Aged; Folic acid; Vitamin $B_{12}$.

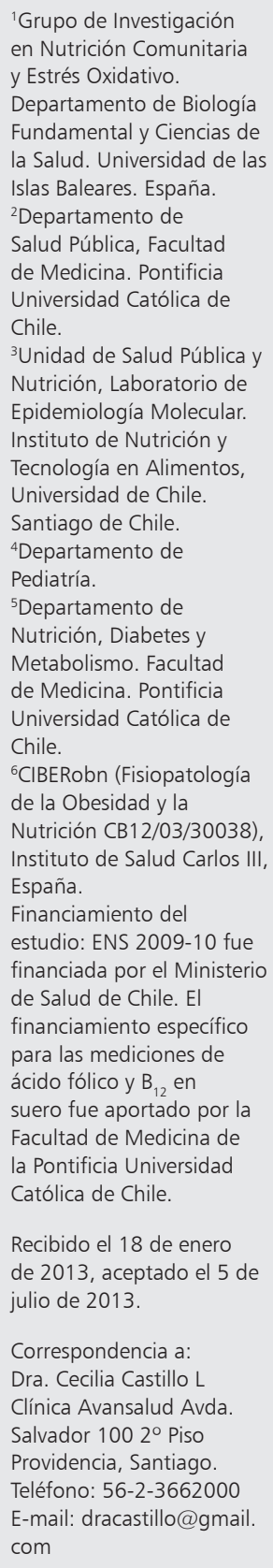

L a fortificación obligatoria de la harina de trigo con ácido fólico se inició en Chile en el año $2002(2-2,4 \mathrm{mg} / \mathrm{kg})$ con el objetivo de reducir el número de recién nacidos con defectos de tubo neural $(\mathrm{DTN})^{1}$. Esta medida ha tenido un enorme impacto en la reducción de estas mal- formaciones congénitas ${ }^{2}$, siendo evaluada como una intervención beneficiosa para la salud pública basada en su costo-efectividad ${ }^{3}$.

Estudios recientes muestran que cerca de 20\% de las muestras de harinas exceden los límites establecidos de fortificación ${ }^{2}$. Estos altos niveles 
de ácido fólico en harina podrían determinar un incremento del folato sérico en niveles superiores a lo estimado, tal como se observa en otros países ${ }^{4,5}$.

Se ha descrito que el incremento de folato sérico tendría un efecto protector en salud cuando existe un déficit previo en algunos tipos de cáncer como colon, mama y páncreas ${ }^{6-8}$. Sin embargo, otros estudios sugieren que niveles elevados podrían determinar efectos adversos en salud también en relación a cáncer ${ }^{9-13}$. En adultos mayores se ha descrito, además, una disminución de la función cognitiva cuando éstos presentan bajos niveles de folato sérico o cuando la elevación está asociada con un déficit de vitamina $\mathrm{B}_{12}{ }^{14}$. Esta información plantea la necesidad de estudiar en Chile la presencia de niveles suprafisiológicos de folato sérico en distintos grupos de población, especialmente en embarazadas, niños y adultos mayores.

El objetivo de este estudio es describir y analizar los niveles séricos de folato y vitamina $B_{12}$ en adultos mayores chilenos provenientes de la Encuesta Nacional de Salud del año 2009-2010 (ENS 2009-10) e identificar potenciales subpoblaciones en riesgo para la salud a causa de estos niveles. Este estudio pretende aportar a la vigilancia epidemiológica nutricional y contribuir a la generación y evaluación de políticas de salud.

\section{Material y Método}

La muestra seleccionada comprende todos los adultos mayores (65 y más años) participantes de la ENS 2009-10 desarrollada entre octubre del año 2009 y septiembre de $2010^{15}$. La base de datos fue proporcionada por el Departamento de Epidemiología del Ministerio de Salud. Ésta corresponde a un estudio de prevalencia realizado en hogares en una muestra nacional, probabilística, estratificada y multietápica de 5.412 personas mayores de 15 años con representatividad nacional, urbana, rural y regional. Para cada individuo se consideró un factor de expansión correspondiente al inverso de la probabilidad de selección del individuo, lo que permite corregir los resultados muestrales considerando la probabilidad desigual de selección de cada entrevistado dado el diseño muestral y la postestratificación demográfica según proyecciones censales a enero de 2010. El grupo de adultos mayores en ENS 2009-10 (1.043 individuos) fue especialmente sobrerrepresentado en el muestreo, con doble probabilidad de selección en el hogar a través de la tabla de Kish con el objetivo de obtener resultados de precisión estadística similar a los otros grupos de población. De éstos, 827 disponían de valores para folato sérico (FS) y 817 para vitamina $\mathrm{B}_{12}$. Los valores extremos $(>62,5 \mu \mathrm{g} / \mathrm{L}$ para folato sérico y $>1.022 \mathrm{pmol} / \mathrm{ml}$ para vitamina $B_{12}$ ) fueron removidos para la descripción de los promedios y medianas nacionales (se excluyeron los valores 3 veces por sobre o bajo el rango intercuartil). Para el cálculo de las prevalencias no se excluyó ningún valor. Los participantes firmaron un consentimiento informado y sus resultados les fueron devueltos con recomendaciones y derivación según correspondía. El protocolo de estudio fue aprobado por el Comité de Ética de Investigación de la Escuela de Medicina de la Pontificia Universidad Católica de Chile ${ }^{20}$.

\section{Laboratorio}

Las muestras de sangre fueron obtenidas por una enfermera entrenada en participantes con ayuno previo. Tecnólogos médicos de los laboratorios regionales de la red de Servicios de Salud centrifugaron y fraccionaron las muestras biológicas recibidas según protocolo, almacenándolas congeladas a $-20^{\circ} \mathrm{C}$ hasta su traslado a Santiago para su análisis en el Laboratorio Central de la Pontificia Universidad Católica de Chile, debiendo cumplir con los controles de calidad interno del laboratorio y lo establecido según acreditación de la Norma Chilena 2.547 e ISO $15.189^{16}$. Noventa y un por ciento de las muestras fueron procesadas antes de $4 \mathrm{~h}$ desde el momento de la punción venosa, previo a lo cual fueron mantenidas refrigeradas a $4^{\circ} \mathrm{C}$. Los niveles séricos de folato y vitamina $B_{12}$ fueron determinados mediante inmunoensayo competitivo por quimioluminiscencia directa (ADVIA Centaur ${ }^{\circledR}$ ).

\section{Análisis estadístico}

La distribución percentilar considerando deciles y los percentiles (P) 5 y 95, así como los promedios de folato $y$ vitamina $B_{12}$ sérico se analizaron según sexo y grupos de edad (65-74 años $y \geq 75$ años). Los sujetos fueron categorizados en cuatro grupos según nivel de folato sérico $(\mu \mathrm{g} / \mathrm{L})$ : $\leq 4,4$ (déficit) ${ }^{17} ; 4,41-20$ (normal) y tres categorías de niveles definidos como suprafisiológicos considerando diferentes puntos de corte: 20,01-25,6 
según Dary $\mathrm{O}^{17} ; 25,601-29$ utilizado por Selhub et al. ${ }^{18} \mathrm{y}>29 \mu \mathrm{g} / \mathrm{L}$ correspondiente al percentil 80 de la distribución de la muestra. Se establecieron tres categorías de nivel sérico de vitamina $\mathrm{B}_{12}(\mathrm{pg} /$ $\mathrm{ml}): \leq 200$ (déficit); 200,1-299,5 (déficit marginal) y $>299,5$ (normal) ${ }^{19}$. Se consideraron tres estratos de nivel educacional (NEDU): educación básica ( $\leq 8$ años), media (8-12 años) y superior $(\geq 12$ años) y dos según zona de residencia (urbana y rural).

Las tasas de prevalencia y medias se calcularon utilizando los factores de expansión conforme al diseño complejo y ajustando la muestra a la demografía chilena a junio de 2010. Se calcularon Odds Ratio ajustados por edad y sexo utilizando regresión logística y también modelos ANCOVA con intervalos de confianza 95\%. Se utilizó el módulo de muestras complejas del programa SPSS v. 17. El nivel de significancia fue definido como $\mathrm{p}<0,05$. Las Tablas muestran prevalencias expandidas, sin embargo, los márgenes muestran el tamaño muestral del estrato.

\section{Resultados}

En la Tabla 1 se muestra las características de la muestra expandida. Cuarenta y dos coma siete por ciento eran hombres, $82,1 \%$ provenía de zonas urbanas y $56,8 \%$ tenía un nivel educacional menor a 8 años de estudios.

En la Tabla 2 y 3 se muestra la distribución percentilar y el promedio del nivel sérico de folato $(\mu \mathrm{g} / \mathrm{L})$ y vitamina $B_{12}(\mathrm{pg} / \mathrm{ml})$ para hombres y mujeres y por grupo de edad. La mediana para ácido fólico en toda la población estudiada fue de $19,5 \mu \mathrm{g} / \mathrm{L}$ y el promedio en hombres y mujeres de $20,1( \pm 0,71)$ y $21,9( \pm 0,8) \mu \mathrm{g} / \mathrm{L}$ respectivamente. En el P5 y P95 se observa un nivel de folato de 8,6 y $9,3 \mu \mathrm{g} / \mathrm{L}$ y de 35,2 y $40,6 \mu \mathrm{g} / \mathrm{L}$ para hombres y mujeres, respectivamente.

Aproximadamente $50 \%$ de la población estudiada presentaba niveles considerados suprafisiológicos (> $20 \mu \mathrm{g} / \mathrm{L})$ alcanzando un nivel de 38,6 $\mu \mathrm{g} / \mathrm{L}$ en el P95 de la población estudiada (Tabla 2). La mediana de vitamina $B_{12}$ para esta población fue de 305 y $330 \mathrm{pg} / \mathrm{ml}$, con un promedio de 325,4 $( \pm 10,84)$ y $346,6( \pm 10,82) \mathrm{pg} / \mathrm{ml}$ en hombres y mujeres respectivamente. Se observa que $5 \%$ de los hombres y mujeres presentaban además un nivel de vitamina $B_{12}$ deficitario $(<200 \mathrm{pg} / \mathrm{ml})$ (Tabla 3$)$.
La distribución porcentual según categorías de folato sérico y vitamina $B_{12}$ se muestran en las Tablas 3 y 4 . Los OR crudo y ajustado según edad y sexo se presentan en la Tabla 5 . Se observa que sólo $0,3 \%$ de la población estudiada presentaba déficit de folatos. Cincuenta y uno coma uno por ciento presentaba niveles normales y $48,6 \%$ presentaba niveles suprafisiológicos $(>20 \mu \mathrm{g} / \mathrm{L})$. Cuando se compara hombres y mujeres con niveles de folato suprafisiológicos $>29 \mu \mathrm{g} / \mathrm{L}$, se observa que los hombres tenían prevalencias de niveles elevados significativamente menores que las mujeres (Tabla 3). Esta tendencia se mantiene al ajustar por todas las variables de control: edad, zona y NEDU (OR 0,47 IC 95\% 0,26-0,84) (Tabla 5). En la Tabla 4 se muestra que $58,3 \%$ de la población estudiada presentaba niveles normales de vitamina $B_{12}$ sérica, 33,6\% déficit marginal $y$ $8,1 \%$ déficit. Se observa que, entre los hombres, $4,5 \%$ del grupo de $65-74$ años y $11 \%$ de los $\geq 75$ años presentaban déficit de vitamina $\mathrm{B}_{12}(<200$ $\mathrm{pg} / \mathrm{L}$ ), mientras que en las mujeres se observa un déficit de 7,1\% en el grupo de 65-74 años y de $12,1 \%$ en las de $\geq 75$ años. Según sexo, 6,6\% de los hombres y $9,2 \%$ de las mujeres presentaban déficit de vitamina $B_{12}$. El análisis bivariado muestra que las mujeres $\geq 75$ años tenían una prevalencia de déficit marginal significativamente menor que los hombres ( $26,2 \%$ vs $45,3 \%, p=0,037)$. Sin embargo, de manera global, no existen diferencias entre hombres y mujeres en cuanto al déficit marginal

\section{Tabla 1. Descripción de la muestra}

\begin{tabular}{|c|c|c|c|}
\hline \multirow[b]{2}{*}{$N(n)$} & \multicolumn{2}{|c|}{$\begin{array}{l}\text { Grupos de edad } \\
\text { (años) }\end{array}$} & \multirow[b]{2}{*}{ Total } \\
\hline & $65-74$ & $\geq 75$ & \\
\hline $\begin{array}{l}\text { Hombres } \\
\text { Muestra expandida } \\
\text { (Muestra real) } \\
\%\end{array}$ & $\begin{array}{c}436.266 \\
(216) \\
45,4\end{array}$ & $\begin{array}{c}233.299 \\
(138) \\
38,5\end{array}$ & $\begin{array}{c}669.565 \\
(354) \\
42,7\end{array}$ \\
\hline $\begin{array}{l}\text { Mujeres } \\
\text { Muestra expandida } \\
\text { (Muestra real) } \\
\%\end{array}$ & $\begin{array}{c}524.799 \\
(304) \\
54,6\end{array}$ & $\begin{array}{c}372.660 \\
(261) \\
61,5\end{array}$ & $\begin{array}{c}8974.59 \\
(565) \\
57,3\end{array}$ \\
\hline Urbano \% & 81,4 & 83,1 & 82,1 \\
\hline $\begin{array}{l}\text { Escolaridad } \\
\quad<8 \text { años \% } \\
8-12 \text { años \% } \\
>12 \text { años \% }\end{array}$ & $\begin{array}{l}52,7 \\
28,3 \\
19,0\end{array}$ & $\begin{array}{r}63,3 \\
30,4 \\
6,3\end{array}$ & $\begin{array}{l}56,8 \\
29,1 \\
14,1\end{array}$ \\
\hline
\end{tabular}


Nivel sérico de folato y vitamina $B_{12}$ en adultos mayores chilenos - C. Castillo-Lancellotti et al

Tabla 2. Distribución percentilar del nivel sérico de folato y vitamina $B_{12}$ en adultos mayores chilenos, ENS 2009-10

\begin{tabular}{|c|c|c|c|c|c|c|c|c|c|c|c|c|}
\hline $\begin{array}{l}\text { Folato sérico } \\
(\mu \mathrm{g} / \mathrm{L})\end{array}$ & P5 & P10 & P20 & P30 & P40 & P50 & P60 & P70 & P80 & P90 & P95 & Promedio \\
\hline \multicolumn{13}{|l|}{ Hombres } \\
\hline $65-74$ & 10,9 & 12,9 & 13,8 & 15,3 & 17,6 & 19,8 & 22,2 & 22,9 & 24,0 & 29,7 & 33,5 & 20,0 \\
\hline$\geq 75$ años & 6,7 & 10,5 & 11,5 & 15,4 & 17,3 & 18,8 & 20,6 & 23,5 & 27,0 & 33,0 & 37,1 & 20,2 \\
\hline Total hombres & 8,6 & 11,1 & 13,6 & 15,3 & 17,3 & 19,5 & 21,5 & 22,9 & 24,4 & 30,5 & 35,2 & 20,1 \\
\hline \multicolumn{13}{|l|}{ Mujeres } \\
\hline $65-74$ & 9,6 & 12,0 & 13,7 & 15,3 & 17,6 & 19,5 & 22,3 & 26,4 & 30,4 & 34,6 & 37,0 & 21,5 \\
\hline$\geq 75$ años & 8,8 & 10,1 & 14,5 & 15,0 & 17,5 & 19,6 & 23,3 & 27,7 & 29,8 & 40,5 & 42,9 & 22,4 \\
\hline Total mujeres & 9,3 & 11,3 & 13,9 & 15 & 17,6 & 19,5 & 22,5 & 26,8 & 30 & 34,9 & 40,6 & 21,9 \\
\hline Total ${ }^{* *}$ & 9,3 & 11,3 & 13,6 & 15,1 & 17,5 & 19,5 & 22,1 & 24 & 29 & 33,6 & 38,6 & 21,2 \\
\hline $\begin{array}{l}\text { Vitamina } B_{12} \\
(p g / m L)\end{array}$ & P5 & P10 & P20 & P30 & P40 & P50 & P60 & P70 & P80 & P90 & P95 & Promedio \\
\hline \multicolumn{13}{|l|}{ Hombres } \\
\hline $65-74$ & 203 & 211 & 236 & 269 & 290 & 311 & 350 & 384 & 432 & 477 & 514 & 333,7 \\
\hline$\geq 75$ años & 141 & 199 & 216 & 229 & 245 & 259 & 310 & 354 & 444 & 480 & 533 & 308,9 \\
\hline Total hombres & 177 & 207 & 229 & 252 & 275 & 305 & 334 & 384 & 432 & 477 & 533 & 325,4 \\
\hline \multicolumn{13}{|l|}{ Mujeres } \\
\hline $65-74$ & 196 & 207 & 224 & 263 & 301 & 330 & 345 & 397 & 455 & 536 & 656 & 358,4 \\
\hline$\geq 75$ años & 173 & 186 & 230 & 263 & 298 & 336 & 371 & 409 & 514 & 595 & 750 & 373,1 \\
\hline Total mujeres & 179 & 198 & 230 & 263 & 299 & 330 & 361 & 404 & 487 & 579 & 701 & 346,6 \\
\hline Total** & 1.179 & 204 & 229 & 259 & 290 & 319 & 345 & 397 & 444 & 532 & 637 & 348,4 \\
\hline
\end{tabular}

**Nivel sérico de folato y vitamina $\mathrm{B}_{12}$ según percentil calculado en el total de la muestra expandida.

de vitamina $\mathrm{B}_{12}$ cuando se desarrolla un análisis bivariado $(29,5 \%$ vs $39,3 \%, \mathrm{p}=0,119)$, así como tampoco, al ajustar por edad, zona y NEDU (OR 1,55 IC 95\% 0,88-2,76) (Tabla 5).

La información sobre el promedio de vitamina $\mathrm{B}_{12}$ sérica según las categorías de folato establecidas se muestra en la Tabla 6. Tanto el análisis de varianza para comparar los niveles de vitamina $\mathrm{B}_{12}$ según niveles de folato, como el análisis de regresión lineal muestran que no hay relación entre los valores de estas variables $(\mathrm{p}=0,828$ y $\mathrm{p}=0,484$ respectivamente). Este resultado se mantiene al ajustar por nivel educacional, sexo, zona y edad.

El porcentaje de adultos mayores en cada una de las categorías de folato según nivel de vitamina $\mathrm{B}_{12}$ se muestra en la Tabla 7. Del total de adultos mayores con déficit de vitamina $\mathrm{B}_{12}, 51,7 \%$ presentaban niveles de folato suprafisiológicos y sólo $0,3 \%$ tenía asociado un déficit de folatos. Del total estudiado, 4,1\% (55.367 adultos mayores) presentaba un nivel suprafisiológico de folato sérico, asociado a un déficit de vitamina $B_{12}$. Por otra parte, de los que presentaban un déficit marginal de vitamina $\mathrm{B}_{12}, 48 \%$ presentaba niveles de folato sérico sobre $20 \mu \mathrm{g} / \mathrm{L}$. El análisis de $\chi^{2}$ sobre esta tabla no muestra relación entre los niveles de folato y vitamina $B_{12}$, no encontrándose una asociación significativa para el modelo $(\mathrm{p}=0,268)$.

\section{Discusión}

Chile no ha desarrollado un sistema de monitoreo de folato sérico después de iniciada la fortificación de alimentos, así como tampoco, de vitamina $B_{12}$. Para efectos de comparación de niveles séricos de vitaminas durante la etapa pre y post-fortificación sólo se encuentran disponibles algunos estudios transversales desarrollados en adultos mayores de la Región Metropolitana ${ }^{19,21,22}$. La ENS 2009-10 es el primer estudio representativo que mide niveles séricos de folato y de vitamina $\mathrm{B}_{12}$ en adultos mayores ${ }^{15}$. 
Nivel sérico de folato y vitamina $B_{12}$ en adultos mayores chilenos - C. Castillo-Lancellotti et al

Tabla 3. Prevalencia de adultos mayores según sexo y grupo de edad con niveles deficitarios, normales y suprafisiológicos de folato sérico, ENS 2009-2010

\begin{tabular}{|c|c|c|c|c|c|c|}
\hline \multirow[b]{2}{*}{ Hombres } & \multicolumn{6}{|c|}{ Folato sérico $(\mu \mathrm{g} / \mathrm{L})$} \\
\hline & $<4,40$ & $4,41-20$ & $20,01-25,6$ & $25,6-29$ & $>29$ & Total \\
\hline \multicolumn{7}{|l|}{ 65-74 años } \\
\hline Muestra real (n) & - & 97 & 46 & 20 & 29 & 192 \\
\hline Muestra expandida (N) & - & 189.337 & 116.986 & 25.235 & 48.822 & 380.380 \\
\hline$\%$ & - & 49,8 & 30,8 & 6,6 & 12,8 & 100,0 \\
\hline \multicolumn{7}{|l|}{$\geq 75$ años } \\
\hline Muestra real (n) & 2 & 56 & 28 & 10 & 25 & 121 \\
\hline Muestra expandida (N) & 373 & 98.371 & 38.751 & 15.761 & 2.832 & 181.577 \\
\hline$\%$ & 0,2 & 54,2 & 21,3 & 8,7 & 15,6 & 100,0 \\
\hline \multicolumn{7}{|l|}{ Total hombres } \\
\hline Muestra (n) & 2 & 153 & 74 & 30 & 54 & 313 \\
\hline Muestra expandida (N) & 373 & 287.707 & 155.738 & 40.996 & 77.144 & 561.958 \\
\hline$\%$ & 0,1 & 51,2 & 27,7 & 7,3 & $13,7^{a}$ & 100,0 \\
\hline Mujeres & $<4,40$ & $4,410-20$ & $20,01-25,6$ & $25,601-29$ & $>29$ & Total \\
\hline \multicolumn{7}{|l|}{ 65-74 años } \\
\hline Muestra real (n) & - & 118 & 69 & 18 & 64 & 269 \\
\hline Muestra expandida (N) & - & 230.378 & 80.316 & 20.732 & 117.303 & 448.729 \\
\hline$\%$ & - & 51,3 & 17,9 & 4,6 & 26,1 & 100 \\
\hline \multicolumn{7}{|l|}{$\geq 75$ años } \\
\hline Muestra real (n) & 4 & 123 & 45 & 22 & 47 & 241 \\
\hline Muestra expandida (n) & 3.287 & 177.371 & 47.467 & 37.746 & 84.279 & 350.150 \\
\hline$\%$ & 0,9 & 50,7 & 13,6 & 10,8 & 24,1 & 100,0 \\
\hline \multicolumn{7}{|l|}{ Total mujeres } \\
\hline Muestra real (n) & 4 & 241 & 114 & 40 & 111 & 510 \\
\hline Muestra expandida (N) & 3.287 & 407.749 & 127.783 & 58.478 & 201.582 & 798.879 \\
\hline$\%$ & 0,4 & 51,0 & 16,0 & 7,3 & $25,2^{a}$ & 100,0 \\
\hline \multicolumn{7}{|l|}{ Total } \\
\hline Muestra real (n) & 6 & 394 & 188 & 70 & 165 & 823 \\
\hline Muestra expandida (N) & 3.660 & 695.456 & 283.521 & 99.474 & 278.725 & 1.360 .837 \\
\hline$\%$ & 0,3 & 51,1 & 20,8 & 7,3 & 20,5 & 100,0 \\
\hline
\end{tabular}

Test $\chi^{2}$ para muestras expandidas Diferencia entre sexos. ${ }^{\mathrm{a}} \mathrm{p}=0,012$.

En un estudio transversal desarrollado en Santiago durante la pre-fortificación en 247 adultos mayores de nivel socioeconómico bajo, se observa un déficit de folato sérico de $55 \%$ en hombres y $33,1 \%$ en mujeres, con promedios de nivel sérico de 3,1 y $3,9 \mu \mathrm{g} / \mathrm{L}$ respectivamente ${ }^{21}$. Aunque no son estudios metodológicamente comparables, los resultados de la ENS muestra niveles mayores de folato sérico, tanto en hombres como en mujeres, (20,1 y $21,9 \mu \mathrm{g} / \mathrm{L}$ respectivamente), mostrando el impacto de la fortificación con ácido fólico. Destaca además en el presente estudio, que el déficit de folatos es prácticamente inexistente.
Si bien en Chile no existe fortificación obligatoria de alimentos con vitamina $B_{12}$, se puede observar que el déficit de vitamina $B_{12}$ es menor $(8,1 \%)$ que en estudios desarrollados en la etapa de prefortificación $(55,1 \%$ en hombres y $30,9 \%$ en mujeres) y postfortificación ${ }^{19,21}$, los cuales consideraban los mismos puntos de corte para definir déficit. Es probable que incorporando otros indicadores más sensibles para la identificación del déficit de vitamina $\mathrm{B}_{12}$ tales como la determinación de ácido metil-malónico y de holotranscobalamina, se pueda mejorar la pesquisa del déficit ${ }^{23}$. A diferencia del estudio de Sánchez et al., en la ENS 2009-10 no 
Nivel sérico de folato y vitamina $B_{12}$ en adultos mayores chilenos - C. Castillo-Lancellotti et al

Tabla 4. Prevalencia de adultos mayores según sexo y grupo de edad que presentan déficit, déficit marginal y niveles normales de vitamina $B_{12}$, ENS 2009-2010

\begin{tabular}{|c|c|c|c|c|}
\hline \multirow[b]{2}{*}{ Hombres } & \multicolumn{4}{|c|}{ Vitamina $B_{12}(p g / m l)$} \\
\hline & $<200$ & $200-299,5$ & $>299,5$ & Total \\
\hline \multicolumn{5}{|l|}{ 65-74 años } \\
\hline Muestra (n) & 24 & 76 & 92 & 192 \\
\hline Muestra expandida (n) & 17.095 & 139.556 & 226.896 & 383.548 \\
\hline$\%$ & 4,5 & 36,4 & 59,2 & 100,0 \\
\hline \multicolumn{5}{|l|}{$\geq 75$ años } \\
\hline Muestra (n) & 18 & 50 & 55 & 123 \\
\hline Muestra expandida (n) & 20.139 & 83.036 & 80.249 & 183.424 \\
\hline$\%$ & 11 & $45,3^{a}$ & 43,8 & 100,0 \\
\hline \multicolumn{5}{|l|}{ Total hombres } \\
\hline Muestra (n) & 42 & 126 & 147 & 315 \\
\hline Muestra expandida (n) & 37.234 & 222.592 & 307.145 & 566.972 \\
\hline$\%$ & 6,6 & 39,3 & 54,2 & 100 \\
\hline Mujeres & $<200$ & $200-299,5$ & $>299,5$ & Total \\
\hline \multicolumn{5}{|l|}{ 65-74 años } \\
\hline Muestra real (n) & 23 & 65 & 176 & 264 \\
\hline Muestra expandida (n) & 31.874 & 143.582 & 274.907 & 450.363 \\
\hline$\%$ & 7,1 & 31,9 & 61,0 & 100,0 \\
\hline \multicolumn{5}{|l|}{$\geq 75$ años } \\
\hline Muestra real (n) & 26 & 63 & 144 & 233 \\
\hline Muestra expandida (n) & 40.328 & 87.547 & 206.301 & 334.176 \\
\hline$\%$ & 12,1 & $26,2^{a}$ & 61,7 & 100,0 \\
\hline \multicolumn{5}{|l|}{ Total mujeres } \\
\hline Muestra real (n) & 49 & 128 & 320 & 497 \\
\hline Muestra expandida (n) & 72.202 & 231.129 & 481.208 & 784.539 \\
\hline$\%$ & 9,2 & 29,5 & 61,3 & 100,0 \\
\hline \multicolumn{5}{|l|}{ Total } \\
\hline Muestra real (n) & 91 & 254 & 467 & 812 \\
\hline Muestra expandida (n) & 10.943 & 453.721 & 788.354 & 1.351 .512 \\
\hline$\%$ & 8,1 & 33,6 & 58,3 & 100,0 \\
\hline
\end{tabular}

Test $\chi^{2}$ para muestras expandidas diferencia entre sexos en $\geq 75$ años, $p=0,045$.

se observan diferencias significativas entre hombres y mujeres después de ajustar por edad, sexo, zona y NEDU ${ }^{19}$. El menor déficit encontrado podría ser explicado por un mayor consumo de alimentos aportadores de vitamina $\mathrm{B}_{12}$, especialmente carnes $^{24}$ o bien a la entrega de alimentos fortificados ${ }^{25}$.

Si bien en el mercado comercial existen diferentes tipos de alimentos fortificados con ácido fólico, la principal fuente la constituyen los elaborados con harina de trigo ${ }^{26}$. Estudios que han evaluado el contenido de ácido fólico en harina muestran que alrededor de 20 a $30 \%$ de las muestras presentan un contenido superior a lo establecido ${ }^{2,27}$.
Esto podría explicar los elevados niveles de folato sérico encontrados en este estudio, donde 48,6\% presenta niveles suprafisiológicos (> $20 \mu \mathrm{g} / \mathrm{L}$ ). Otros países que fortifican en forma obligatoria, también muestran incrementos del folato sérico, especialmente en adultos mayores ${ }^{28}$. Se observa además, que $13,7 \%$ de los hombres y $25,2 \%$ de las mujeres presentan niveles especialmente elevados $(>29 \mu \mathrm{g} / \mathrm{L})$. Es probable que estos adultos pudieran corresponder a un subgrupo de población con mayor consumo de alimentos elaborados con harina de trigo, pero también podría asociarse a ingesta de multivitamínicos ${ }^{29}$. 
Nivel sérico de folato y vitamina $B_{12}$ en adultos mayores chilenos - C. Castillo-Lancellotti et al

Tabla 5. Odds ratio de prevalencia para niveles normales, deficitarios y suprafisiológicos de ácido fólico según edad, sexo y nivel educacional, ENS 2009-2010

\begin{tabular}{|c|c|c|c|c|c|}
\hline Folato sérico $(\mu \mathrm{g} / \mathrm{L})$ & & OR Crudo & IC $95 \%$ & OR Ajustadoa & IC $95 \%$ \\
\hline \multirow[t]{2}{*}{$<4$} & Edad & - & & - & - \\
\hline & Sexo & 0,16 & $0,018-1,47$ & - & - \\
\hline \multirow[t]{2}{*}{$4,41-20$} & Edad & 1,05 & $0,64-1,73$ & 1,07 & $0,66-1,73$ \\
\hline & Sexo & 1,01 & $0,59-1,72$ & 1,01 & $0,60-1,71$ \\
\hline \multirow[t]{2}{*}{$>20$} & Edad & 0,93 & $0,56-1,53$ & 0,91 & $0,56-1,47$ \\
\hline & Sexo & 1,01 & $0,59-1,72$ & 1,0 & $0,59-1,68$ \\
\hline \multirow[t]{2}{*}{$>25,6$} & Edad & 1,32 & $0,77-2,26$ & 1,15 & $0,68-1,94$ \\
\hline & Sexo & 0,55 & $0,32-0,96$ & 0,57 & $0,34-0,997$ \\
\hline \multirow[t]{2}{*}{$>29$} & Edad & 1,07 & $0,59-1,95$ & 0,93 & $0,52-1,69$ \\
\hline & Sexo & 0,47 & $0,26-0,86$ & 0,47 & $0,26-0,84$ \\
\hline \multicolumn{6}{|l|}{ Vitamina $B_{12}(p g / m l)$} \\
\hline \multirow[t]{2}{*}{$<200$} & Edad & 2,12 & $0,98-4,06$ & 1,84 & $0,82-4,13$ \\
\hline & Sexo & 0,69 & $0,32-1,49$ & 0,82 & $0,39-1,71$ \\
\hline \multirow[t]{2}{*}{$200-299,5$} & Edad & 0,96 & $0,57-1,62$ & 1,0 & $0,60-1,69$ \\
\hline & Sexo & 1,55 & $0,89-2,68$ & 1,55 & $0,88-2,76$ \\
\hline
\end{tabular}

aGrupos de referencia para el cálculo de odds ratios ajustados: mujeres, edad $\geq 75$ años; zona urbano; nivel educacional alto: $>12$ años estudios.

Tabla 6. Nivel sérico de vitamina $B_{12}{ }^{*}$ según categorías de folato * en adultos mayores, ENS 2009-2010

\begin{tabular}{|lccccc|}
\hline & \multicolumn{4}{c}{ Nivel sérico de ácido fólico $(\boldsymbol{\mu g} / \mathbf{L})$} \\
& $\mathbf{4 , 4 1}$ & $\mathbf{4 , 4 1 - 2 0}$ & $\mathbf{2 0 , 0 1 - 2 5 , 6}$ & $\mathbf{2 5 , 6 0 1 - 2 9}$ & $>\mathbf{2 9}$ \\
$\begin{array}{l}\text { Nivel sérico* } \\
\text { vitamina } B_{12}(\mathrm{pg} / \mathrm{ml})^{*}\end{array}$ & 421,8 & 391,4 & 439,3 & 364,2 & 383,9 \\
IC 95\% & $332,4-511,1$ & $351,2-471,5$ & $308,6-570,1$ & $281,1-443,3$ & $343,6-424,3$ \\
Error típico & 45,5 & 20,4 & 66,6 & 42,3 & 20,5 \\
\hline
\end{tabular}

*promedio. ANOVA: $p=0,484$ NS.

Tabla 7. Distribución de adultos mayores según nivel de sérico de folato y niveles séricos de vitamina $B_{12}$, ENS 2009-2010

\begin{tabular}{|c|c|c|c|c|c|c|c|c|}
\hline \multirow{3}{*}{$\begin{array}{l}\text { Folato } \\
(\mu \mathrm{g} / \mathrm{L})\end{array}$} & \multicolumn{8}{|c|}{ Vitamina $B_{12}(p g / m l)$} \\
\hline & \multicolumn{2}{|c|}{$<200$} & \multicolumn{2}{|c|}{$200-299,5$} & \multicolumn{2}{|l|}{$>299,5$} & \multicolumn{2}{|l|}{ Total } \\
\hline & $\mathbf{N}(\mathbf{n}) *$ & $\%$ & $\mathbf{N}(\mathbf{n}) *$ & $\%$ & $\mathbf{N}(\mathbf{n}) *$ & $\%$ & $\mathbf{N}(\mathbf{n})^{*}$ & $\%$ \\
\hline$<4,41$ & $357(1)$ & 0,3 & $16(1)$ & 0,0 & $3.287(4)$ & 0,4 & $3.660(6)$ & 0,3 \\
\hline $4,41-20$ & $51.323(37)$ & 47,9 & $235.940(122)$ & 52,0 & $391.196(223)$ & 50,5 & $678.459(382)$ & 50,8 \\
\hline $20,01-25,6$ & $15.598(19)$ & 14,6 & $99.512(64)$ & 21,9 & $159.669(97)$ & 20,6 & $274.779(180)$ & 20,6 \\
\hline $25,601-29$ & $11.830(11)$ & 11,0 & $50.443(22)$ & 11,1 & $37.201(37)$ & 4,8 & $99.474(70)$ & 7,5 \\
\hline$>29$ & $27.949(22)$ & 26,1 & $67.483(44)$ & 14,9 & $183.293(99)$ & 23,7 & $278.725(165)$ & 20,9 \\
\hline Total & $107.958(90)$ & 100 & $453.393(253)$ & 100 & $774.647(460)$ & 100 & $1.335 .097(803)$ & 100 \\
\hline
\end{tabular}

${ }^{*} N=$ Muestra expandida. $(n)=$ muestra real. Test $\chi^{2}$ para muestras expandidas $p=0,268$ NS. 
El análisis adicional de la base de datos de medicamentos de la ENS 2009-10 muestra que $4,7 \%$ y $3,2 \%$ de los adultos mayores estudiados consumen suplementos vitamínicos conteniendo ácido fólico y vitamina $B_{12}$, siendo su consumo significativamente mayor en aquellos con niveles de folato sérico $>20 \mu \mathrm{g} / \mathrm{L}$. Por otra parte, del total que no usan suplementos, $49,5 \%$ tienen niveles de folatos considerados suprafisiológicos, lo cual sugiere vigilar la política de fortificación más estrechamente.

En el presente estudio sólo se determinó folato sérico total, sin embargo, considerando que cerca de la mitad de los adultos mayores presenta niveles suprafisiológicos, sería recomendable determinar la presencia de ácido fólico libre considerando las implicancias en salud. Se ha descrito que cuando los niveles de folato sérico son elevados, un porcentaje variable corresponde a ácido fólico sin metabolizar $^{30}$. Su presencia podría ser explicada por diversos factores tales como ingestas dietarias superiores a $400 \mu \mathrm{g} / \mathrm{día}^{31}$, limitada conversión a tetrahidrofolato derivada de la rápida saturación de la enzima dihidrofolato reductasa (DHFR) ${ }^{32}$ y por la existencia de polimorfismos, que determinan una menor actividad enzimática ${ }^{33}$. Es decir, la presencia de ácido fólico circulante podría ser más relevante en ciertas poblaciones quienes responderían de manera distinta dependiendo del tiempo y la dosis ingerida. Algunos estudios, pero no todos ${ }^{6}$, sugieren ${ }^{11,33-35}$ que niveles elevados de folato sérico, especialmente como ácido fólico, podría promover el crecimiento de algunos cánceres o de lesiones preexistentes. Un metanálisis reciente, con seguimiento durante cinco años, no mostró una mayor incidencia ${ }^{36}$. Estudios recientes describen además, un aumento en el deterioro en el sistema nervioso central, especialmente asociado a deficiencia de vitamina $\mathrm{B}_{12}{ }^{12}$. Al parecer existiría una interacción clave entre vitamina $B_{12} y$ folato en la síntesis de metionina desde la homocisteína a través de la enzima metionina sintetasa, en los cuales el 5-metil-tetrahidrofolato y la vitamina $B_{12}$ actúan como cofactores. Se especula que el exceso de ácido fólico y sus metabolitos actuarían como aceptores de electrones (oxidantes), exacerbando la deficiencia de vitamina $B_{12}$ de manera semejante a lo observado en la exposición a óxido nitroso ${ }^{37}$. En algunos estudios se ha descrito que en individuos con mayores niveles de folato sérico se exacerbaría el déficit de vitamina $\mathrm{B}_{12}{ }^{18,38}$. En nuestro estudio, al igual que en el estudio de Mills JL et al. ${ }^{39}$, no se observa diferencias significativas en los niveles de vitamina $\mathrm{B}_{12}$ considerando diferentes categorías de folatos.

En resumen, el déficit de folatos observado en Chile es mínimo, sin embargo, un porcentaje importante de la población presenta niveles suprafisiológicos sugiriendo la conveniencia de establecer un sistema de vigilancia para evaluar y limitar los probables riesgos en salud, adecuando la fortificación de alimentos.

\section{Referencias}

1. Gobierno de Chile. Ministerio de Salud. Programas de Fortificación de alimentos. Disponible en http://www. minsal.gob.cl/portal/url/page/minsalcl/g_proteccion/g_ alimentos/prot_fortificacion.html [Consultado el 6 de mayo de 2013].

2. Castillo-Lancellotti C, Tur JA, Uauy R. Impact of folic acid fortification of flour on neural tube defects: a systematic review. Public Health Nutr 2012; 31: 1-11.

3. Llanos A, Hertrampf E, Cortés F, Pardo A, Grosse SD, Uauy R. Cost-effectiveness of a folic acid fortification program in Chile. Health Policy 2007; 83 (2-3): 295303.

4. Pfeiffer CM, Hughes JP, Lacher DA, Bailey Rl, Berry RJ, Zhang $\mathrm{M}$, et al. Estimation of trends in serum and RBC folate in the U.S. population from pre-to postfortification using assay-adjusted data from the NHANES 19882010. J Nutr 2012; 142 (5): 886-93.

5. Ray JG, Vermeulen MJ, Boss SC, Cole DE. Increased red cell folate concentrations in women of reproductive age after Canadian folic acid food fortification. Epidemiology 2002; 13 (2): 238-40.

6. Giovannucci E, Stampfer MJ, Colditz GA, Hunter DJ, Fuchs C, Rosner BA, et al. Multivitamin use, folate, and colon cancer in women in the Nurses' Health Study. Ann Intern Med 1998; 129 (7): 517-24.

7. Shrubsole MJ, Jin F, Dai Q, Shu XO, Potter JD, Hebert Jr, et al. Dietary folate intake and breast cancer risk: results from the Shanghai Breast Cancer Study. Cancer Res 2001; 61 (19): 7136-41.

8. Chuang SC, Stolzenberg-Solomon R, Ueland PM, Vollset SE, Midttun $\varnothing$, Olsen A, et al. A U-shaped relationship between plasma folate and pancreatic cancer risk in the European Prospective Investigation into Cancer and Nutrition. Eur J Cancer 2011; 47 (12): 1808-16.

9. Kim YI Folate and colorrectal cancer: An evidence-base critical review. Mol Nutr Food Res 2007; 51 (3): 267-92.

10. Stolzenberg-Solomon RZ, Chang SC, Leitzmann MF, 
Johnson KA, Johnson C, Buys SS, et al. Folate intake, alcohol use, and postmenopausal breast cancer risk in the Prostate, Lung, Colorectal, and Ovarian Cancer Screening Trial. Am J Clin Nutr 2006; 83 (4): 895-904.

11. Tomaszewski JJ, Cummings JL, Parwani AV, Dhir R, Mason JB, Nelson JB, et al. Increased cancer cell proliferation in prostate cancer patients with high levels of serum folate. Prostate 2011; 71 (12): 1287-93.

12. Ly A, Hoyt L, Crowell J, Kim YI. Folate and DNA methylation. Antioxid Redox Signal 2012; 17 (2): 302-26.

13. Sweeney MR, McPartlin J, Scott J. Folic acid fortification and public health: report on threshold doses above which unmetabolised folic acid appear in serum. BMC Public Health 2007; 7: 41.

14. Morris MS, Jacques PF, Rosenberg IH, Selhub J. Circulating unmetabolized folic acid and 5-methyltetrahydrofolate in relation to anemia, macrocytosis, and cognitive test performance in American seniors. Am J Clin Nutr 2010; 91 (6): 1733-44.

15. Gobierno de Chile Ministerio de Salud. Encuesta Nacional de Salud ENS 2009-2010 Disponible en www. redsalud.gov.cl [Consultado el 6 de septiembre de 2012].

16. Pontificia Universidad Católica de Chile. Políticas de Calidad de Laboratorios Clínicos. Disponible en http:// redsalud.uc.cl/link.cgi/MS/Laboratorios/Somos/2626 [Consultado el 6 de septiembre de 2012].

17. Dary O. Nutritional interpretation of folic acid interventions. Nutr Rev 2009; 67 (4): 235-44.

18. Morris MS, Jacques PF, Rosenberg IH, Selhub J. Folate and vitamin B-12 status in relation to anemia, macrocytosis, and cognitive impairment in older Americans in the age of folic acid fortification. Am J Clin Nutr 2007; 85 (1): 193-200.

19. Sánchez H, Albala C, Hertrampf E, Verdugo R, Lavados $\mathrm{M}$, Castillo JL, et al. [Prevalence of vitamin B-12 deficiency in older adults]. Rev Med Chile 2010; 138 (1): 44-52.

20. Pappas G, Hyder A. Exploring ethical considerations for the use of biological and physiological markers in population-based surveys in less developed countries. Global Health 2005; 1: 16. doi: 10.1186/1744-8603-116.

21. Olivares M, Hertrampf E, Capurro MT, Wegner D. Prevalence of anemia in elderly subjects living at home: role of micronutrient deficiency and inflammation. Eur J Clin Nutr 2000; 54 (11): 834-9.

22. Hirsch S, de La Maza P, Barrera G, Gattás V, Petermann $\mathrm{M}$, Bunout D. The Chilean flour folic acid fortification program reduces serum homocysteine levels and masks vitamin $B_{12}$ deficiency in elderly people. J Nutr 2002; 132 (2): 289-91.
23. Green R. Indicators for assessing folate and vitamin $B_{12}$ status and for monitoring the efficacy of intervention strategies. Am J Clin Nutr 2011; 94 (2): 666S-72S.

24. Crovetto M, Uauy R. [Changes in processed food expenditure in the population of Metropolitan Santiago in the last twenty years]. Rev Med Chile 2012; 140 (3): 305-12.

25. Gobierno de Chile. Ministerio de Salud. Programa de Alimentación Complementaria del Adulto Mayor. PACAM. Disponible en: http://www.redsalud.gov.cl/portal/ url/page/minsalcl/g_proteccion/g_alimentos/prot_pan. html [Consultado el 23 agosto de 2012].

26. Olivares S, Bustos N, Lera L, Zelada ME. Estado nutricional, consumo de alimentos y actividad física en escolares mujeres de diferente nivel socioeconómico de Santiago de Chile. Rev Med Chile 2007; 135: 71-8.

27. Castillo C, Tur JA, Uauy R. Fortificación de la harina de trigo con ácido fólico en Chile. Consecuencias no intencionadas. Rev Med Chile 2010; 138 (7): 832-40.

28. Vanderwall CM, Tangney CC, Kwasny MJ, Gustashaw KA. Examination of circulating folate levels as a reflection of folate intakes among older adult supplement users and nonusers in the National Health and Nutrition Examination Survey 2003-2004. J Acad Nutr Diet 2012; 112 (2): 285-90.

29. Boilson A, Staines A, Kelleher CC, Daly L, Shirley I, Shrivastava A, et al. Unmetabolized folic acid prevalence is widespread in the older Irish population despite the lack of a mandatory fortification program. Am J Clin Nutr 2012; 96 (3): 613-21.

30. Sweeney Mr, MC Partlin J, Scott J. Folic acid fortification and public health: report on threshold doses above which unmetabolised folic acid appear in serum. BMC Public Health 2007; 7: 41.

31. Bailey SW, Ayling JE. The extremely slow and variable activity of dihydrofolate reductase in human liver and its implications for high folic acid intake. Proc Natl Acad Sci USA 2009; 106 (36): 15424-9.

32. Thomas P, Fenech M. Methylenetetrahydrofolate reductase, common polymorphisms, and relation to disease. Vitam Horm 2008; 79: 375-9.

33. Cole BF, Baron JA, Sandler RS, Haile RW, Ahnen DJ, Bresalier RS, et al. Folic acid for the prevention of colorectaladenomas: a randomized clinical trial. JAMA 2007; 297: 2351-9.

34. Mason JB, Dickstein A, Jacques PF, Haggarty P, Selhub J, Dallal G, et al. A temporal association between folic acid fortification and an increase in colorectal cancer rates may be illuminating important biological principles: a hypothesis. Cancer Epidemiol Biomarkers Prev 2007; 16: 1325-9. 
Nivel sérico de folato y vitamina $B_{12}$ en adultos mayores chilenos - C. Castillo-Lancellotti et al

35. Hirsch S, Sánchez H, Albala C, Maza MP, Barrera G, Leiva L, et al. Colon cancer in Chile before and after the start of the flour fortification program with folic acid. Eur J Gastroenterol Hepatol 2009; 21: 436-9.

36. Vollset SE, Clarke R, Lewington S, Ebbing M, Halsey J, Lonn E, et al. Effects of folic acid supplementation on overall and site-specific cancer incidence during the randomised trials: meta-analyses of data on 50,000 individuals. Lancet 2013: S0140-6736 (12) 62001-7.

37. Reynolds EH. Benefits and risks of folic acid to the ner- vous system. J Neurol Neurosurg Psychiatry 2002; 72: 567-71.

38. Selhub J, Morris MS, Jacques PF, Rosenberg IH. Folatevitamin B-12 interaction in relation to cognitive impairment, anemia, and biochemical indicators of vitamin B-12 deficiency. Am J Clin Nutr 2009; 89: 702S-6S.

39. Mills JL, Carter TC, Scott JM, Troendle JF, Gibney ER, Shane B, et al. Do high blood folate concentrations exacerbate metabolic abnormalities in people with low vitamin B-12 status? Am J Clin Nutr 2011; 94 (2): 495500 . 